\title{
Biweekly docetaxel, cisplatin, and 5-fluorouracil (DCF) chemotherapy for advanced esophageal squamous cell carcinoma: a phase I dose-escalation study
}

\author{
Yoshihiro Tanaka $\cdot$ Kazuhiro Yoshida • \\ Yuichi Sanada · Shinji Osada • Kazuya Yamaguchi • \\ Takao Takahashi
}

Received: 22 May 2010/Accepted: 24 August 2010/Published online: 28 September 2010

(C) The Author(s) 2010. This article is published with open access at Springerlink.com

\begin{abstract}
Background and purpose The optimal chemotherapeutic protocol for the treatment of esophageal cancer has not yet been established. A dose-escalation study of docetaxel combined with cisplatin and 5-fluorouracil (5-FU) was performed to determine the optimal dose in patients with advanced esophageal squamous cell carcinoma.

Patients and method We studied a total of 18 patients who had previously untreated thoracic esophageal squamous cell carcinoma with $\mathrm{T} 4$ tumors and/or metastasis. The patients received an infusion of docetaxel at different dose levels (levels 1, 2, 3: 30, 35, $40 \mathrm{mg} / \mathrm{m}^{2}$, respectively) and an infusion of cisplatin $\left(40 \mathrm{mg} / \mathrm{m}^{2}\right)$ on days 1 and 15 plus a continuous infusion of 5 -FU $\left(400 \mathrm{mg} / \mathrm{m}^{2} /\right.$ day $)$ on days $1-5$ and $15-19$.

Results Dose-limiting toxicities (DLT) included febrile neutropenia and leukopenia. DLT occurred in 2 of 6 patients at level 1, 2 and in 3 of 6 patients at level 3. The response rate was $88.9 \%$, including a complete response rate of $33.3 \%$.

Conclusions To minimize toxicity and maximize dose intensity, we elected to investigate a biweekly regimen. The maximum tolerated dose was level 3 , and the recommended dose was determined to be docetaxel $35 \mathrm{mg} / \mathrm{m}^{2}$ with cisplatin $40 \mathrm{mg} / \mathrm{m}^{2}$ plus 5 -FU $400 \mathrm{mg} / \mathrm{m}^{2}$, administered biweekly. This regimen was tolerable and highly active. A phase II study has been started.
\end{abstract}

Y. Tanaka $(\bowtie) \cdot$ K. Yoshida $\cdot$ Y. Sanada $\cdot$ S. Osada

K. Yamaguchi · T. Takahashi

Department of Surgical Oncology,

Gifu Graduate School of Medicine,

1-1, Yanagido, Gifu city, Gifu 501-1194, Japan

e-mail: yoshihirotana11@hotmail.com
Keywords Chemotherapy - Docetaxel - Cisplatin · 5-Fluorouracil · Esophageal carcinoma

\section{Introduction}

Locally advanced esophageal carcinoma is often refractory to current therapeutic approaches, and its prognosis is grim $[1,2]$. Patients with unresectable or inoperable disease are usually treated with chemotherapy. Although various chemotherapy regimens are available, esophageal cancer carries a very poor prognosis, with a mean survival time of less than 8.1 months with current chemotherapies used singly or in combination with 5-fluorouracil (5-FU), vindesine, mitomycin, docetaxel, paclitaxel, cisplatin, irinotecan, vinorelbine, or capecitabine [3]. Fluorouracil and cisplatin combination therapy (FP) is regarded as standard [4], for which the median survival time is reported to be 9.2 months for responders and 5.3 months for nonresponders [5]. The response rates reported with FP range from 35 to $40 \%$, whereas the 2-year survival rates of patients with locally advanced esophageal cancer range from 8 to $55 \%$, with a mean $27 \%$ [6-8]. To improve both local and distant tumor control in patients with esophageal carcinoma, new therapeutic regimens must be developed. Recently, faborite antitumor effects of combination therapy with fluorouracil and taxen were reported. Docetaxel/S-1 combination is highly active and well tolerated in advanced or recurrent gastric cancer [9], and those synergistic antitumor activity has been fully elucidated [10].

As for advanced esophageal carcinoma, combination of docetaxel and 5-FU with concurrent radiotherapy had good efficacy [11].

The taxanes have been shown to be active agents in the treatment of esophageal carcinoma. Many studies have 
shown that taxanes have significant activity in patients with locally advanced and metastatic esophageal carcinomas [12].

Docetaxel (TXT), cisplatin (CDDP), and 5-FU have shown activity in upper gastrointestinal malignancies with different mechanisms. In Europe, TXT, CDDP, and 5-FU (DCF) combination therapy has been commonly used in patients with advanced gastric cancer. In a report from the V325 study group, advanced gastric or gastroesophageal junction cancer patients receiving DCF not only had statistically improved overall survival and time to tumor progression, but they also had better preservation of quality of life compared with patients receiving FP therapy [13].

The addition of TXT to CDDP plus 5-FU is expected to improve treatment outcomes for patients with esophageal carcinoma.

When we were using combination therapy for advanced esophageal squamous cell carcinoma based on the regimen established by Ajani et al., as a pilot study, marked myelosuppression occurred in all patients.

We therefore conducted a phase I clinical trial of DCF in patients with advanced thoracic esophageal carcinoma with T4 tumors and/or M1 staging with dividedly administration of each drug into two blocks per one course. This phase I, dose-escalation study of TXT, CDDP, and 5-FU was conducted at the Department of Surgical Oncology, Gifu University School of Medicine. The goal of this trial was to determine the recommended dose (RD) for use in phase II trials on the basis of the maximum tolerated dose (MTD) and dose-limiting toxicity (DLT). Secondary objectives were treatment-related toxicity and efficacy.

\section{Patients and methods}

\section{Patient eligibility criteria}

To be eligible for the study, patients had to be at least 18 years of age at the time of registration and have histologically or cytologically confirmed squamous cell carcinoma (SCC), which was surgically unresectable or recurrent. They also had to have an EOCG performance status of 0,1 , or 2 , a life expectancy of $>12$ weeks, and adequate liver, bone marrow, renal and cardiovascular function (serum bilirubin $\leq 1.5 \mathrm{mg} / \mathrm{dl}$; neutrophil count $\geqq 1,500 / \mathrm{mm}^{3}$; serum aspartate aminotransferase (AST) and alanine aminotransferase (ALT) $\leq$ twice the upper limit of normal range; platelet count $\geq 10 \times 10^{4} / \mathrm{mm}^{3}$; hemoglobin $\geq 8.0 \mathrm{~g} / \mathrm{dl}$; and creatinine $\leq 1.2 \mathrm{mg} / \mathrm{dl}$ (or creatinine clearance $>60 \mathrm{ml} / \mathrm{min}$ ). Patients previously treated with taxane therapy for recurrent disease or irradiation to major bone areas were excluded from the study. Finally, the last chemotherapeutic treatment had to be at least 4 weeks before trial enrollment. The major exclusion criteria included serious concomitant illness, symptomatic infectious disease, severe drug allergy, symptomatic peripheral neuropathy, or uncontrolled diabetes mellitus. All the participants had to sign an informed consent form approved by the Ethical Committee of Gifu University Hospital before study entry. Finally, 18 patients were entered, and all patients had fully undergone DCF therapy.

\section{Study design}

The primary objectives of this phase I study were to determine the MTD and toxicity of escalating doses of TXT combined with a fixed dose of CDDP and 5-FU in patients with advanced esophageal carcinoma. The secondary objective of the study was to obtain preliminary data regarding the clinical response.

At least three patients were entered at each TXT dose level. There was no dose-escalation within individual patients or within a dose level. All three patients at a given dose level completed the first two cycles of treatment without DLT before further patients were enrolled in the next dose level. In the absence of DLT, the next dose level was explored. Doses were escalated in sequential groups of three patients until the MTD was established or the highest intended dose levels were reached. If any of the three patients experienced DLT, an additional 3 patients were treated at the same dose level. If three or more of the six patients at a given dose level experienced DLT, the dose level was defined as the MTD. The level one step below was set as the RD for further evaluation in a phase II study.

Treatment plan

Patients received TXT diluted in $250 \mathrm{ml}$ of normal saline at the assigned dose. It was infused intravenously (iv) over $2 \mathrm{~h}$, and a maintenance infusion was given for the next $2 \mathrm{~h}$. Then CDDP was prepared in normal saline at a dose of $40 \mathrm{mg} / \mathrm{m}^{2}$ and administered over $2 \mathrm{~h}$ on day 1.5 -FU was prepared in normal saline at a dose of $400 \mathrm{mg} / \mathrm{m}^{2}$ and administered continuously on days $1-5$. TXT and CDDP were given on days 1 and 15, and 5-FU was given on days $1-5$ and $15-19$ of every 28 -day cycle. The dose-escalation

Table 1 Dose-escalation scheme

\begin{tabular}{llll}
\hline Dose level & $\begin{array}{l}\text { Docetaxel } \\
\left(\mathrm{mg} / \mathrm{m}^{2}\right)\end{array}$ & $\begin{array}{l}\text { Cisplatin } \\
\left(\mathrm{mg} / \mathrm{m}^{2}\right)\end{array}$ & $\begin{array}{l}5 \mathrm{FU} \\
\left(\mathrm{mg} / \mathrm{m}^{2}\right)\end{array}$ \\
\hline 1 & 30 & 40 & 400 \\
2 & 35 & 40 & 400 \\
3 & 40 & 40 & 400 \\
\hline
\end{tabular}


scheme is described in Table 1. The initial dose of TXT was $30 \mathrm{mg} / \mathrm{m}^{2}$ (dose level I), and this was increased up to a maximum of $40 \mathrm{mg} / \mathrm{m}^{2}$ in $5 \mathrm{mg} / \mathrm{m}^{2}$ steps.

Supportive therapy for treatment and prophylaxis for expected side effects was performed. All patients were premedicated with granisetron $2 \mathrm{mg}$ iv. Hypersensitivity reactions were treated with prophylactic use of dexamethasone $8 \mathrm{mg}$ iv, which was infused $1 \mathrm{~h}$ prior to the administration of TXT. Further dexamethasone was prescribed at a dose of $4 \mathrm{mg}$ orally for 2 days after administration of TXT to reduce the risk of hypersensitivity reaction and fluid retention. Diuretics were added at the discretion of the treating physician. Appropriate hydration was given before and after the cisplatin infusion. Antiemetics were recommended on subsequent days as needed.

To avoid severe mucositis, L-glutamine $8 \mathrm{~g}$ was administrated for all patients.

\section{Patient monitoring and response criteria}

All patients underwent complete staging procedures to document disease extent, including EOCG performance status, medical history, and physical examination. Prestudy laboratory evaluation including a complete blood cell count; serum electrolytes; urea; creatinine and 24-h creatinine clearance; bilirubin; alkaline phosphatase and transaminases; CEA, SCC, CA19-9 and CYFRA measurements, and electrocardiogram was obtained within 1 week before initiation of treatment and at the start of each treatment cycle. Baseline computed tomography (CT) or magnetic resonance imaging (MRI) scans and positron emission tomography-CT were performed within 4 weeks prior to study entry. All patients had a complete blood count taken every week during therapy. Levels of electrolytes, serum creatinine, transaminases, alkaline phosphatase and bilirubin, and plasma urea were measured every 2 weeks during therapy. Medical history including toxicity, physical examination, and toxicity assessment was graded every 2 weeks during the study according to the Common Terminology Criteria for Adverse Events (v3.0). Tumor measurements were made from radiographic films or scans taken to document treatment response during therapy and were repeated at every second cycle of treatment or sooner if the patient appeared to show disease progression. Tumor response was assessed based on the Response Evaluation Criteria in Solid Tumors (RECIST) criteria [14]. The response status of measurable lesions was evaluated by a barium meal study, endoscopy, ultrasonography and CT, or MRI.

A complete response was defined as complete disappearance of all clinically detectable malignant diseases. A partial response was defined as a $\geq 30 \%$ decrease in the sum of the perpendicular diameters of all measurable lesions lasting at least 4 weeks. Progressive disease was defined as $\geq 20 \%$ increase in the sum of products of measurable lesions over the smallest sum observed or appearance of new lesions. Stable disease did not qualify as complete response, partial response, or progressive disease.

Definition of dose-limiting toxicity and criteria for dose modifications

Toxicity was evaluated and scored according to the Common Terminology Criteria for Adverse Events (v3.0). DLT was defined as febrile grade 3 neutropenia, grade 4 neutropenia lasting $>7$ days, grade 3 leucopenia, grade 4 thrombocytopenia, or grade 3 thrombocytopenia with bleeding tendency, or any grade 3-4 non-hematological toxicity with the exceptions of nausea, vomiting, diarrhea, and alopecia. If hematological toxicity $\geq$ grade 3 occurred, administration of the therapy was delayed until the platelet count was $10 \times 10^{4} / \mathrm{mm}^{3}$ and absolute neutrophils were $\geq 2 \times 10^{3}$. If gastrointestinal toxicity $\geq$ grade 3 occurred, the administration of chemotherapy was delayed until the optimum dose could be tolerated. Treatment was repeated every 4 weeks or as soon as a patient had recovered from the toxicity of the previous chemotherapy. However, if toxicities persisted for more than 2 weeks following the time of planned treatment, the patient was removed from the study. A delay longer than 2 weeks in administering the second cycle of therapy was also considered a DLT. Dose modifications for the next dose were based on the most severe toxicity observed since the previous treatment course. Treatment was interrupted until toxicity resolved to $\leq$ grade 1 .

\section{Results}

\section{Patient characteristics}

Between October 2008 and December 2009, 18 patients were entered into the present study. Demographic and clinical characteristics of the study population are summarized in Table 2. Three dose levels were evaluated. The patients received a total of 70 courses of chemotherapy, with the median number of courses administered per patient being 3.9 (range, 2-6). All patients in the study had locally advanced esophageal carcinoma or metastatic lesions. Median patient age was 62.8 years (range, 50-79 years). All patients had an ECOG performance status (PS) of 0-1. Histology showed moderately welldifferentiated carcinoma in $38.9 \%$ of the patients and poorly differentiated carcinoma in $16.7 \%$. 
Table 2 Characteristics of patients

\begin{tabular}{|c|c|c|}
\hline $\begin{array}{l}\text { Characteristics } \\
\text { No. of patients }\end{array}$ & $\begin{array}{l}\text { No. } \\
18\end{array}$ & $\%$ \\
\hline \multicolumn{3}{|l|}{ Age, years } \\
\hline Median & 62.8 & \\
\hline Range & $50-79$ & \\
\hline \multicolumn{3}{|l|}{ Sex } \\
\hline Males & 15 & 83.3 \\
\hline Females & 3 & 16.7 \\
\hline \multicolumn{3}{|l|}{ Performance status } \\
\hline $0-1$ & 18 & 100 \\
\hline 2 & 0 & 0 \\
\hline \multicolumn{3}{|l|}{ Disease status } \\
\hline Locally advanced & 9 & 50 \\
\hline Locally advanced and metastatic & 9 & 50 \\
\hline \multicolumn{3}{|l|}{ Stage of disease } \\
\hline T3N1M0 & 2 & 11.1 \\
\hline $\mathrm{T} 3 \mathrm{~N} 2 \mathrm{M} 0$ & 4 & 22.2 \\
\hline T3N4M0 & 3 & 16.7 \\
\hline T3N4M1 & 3 & 16.7 \\
\hline T4N1M1 & 4 & 22.2 \\
\hline T4N2M1 & 2 & 11.1 \\
\hline \multicolumn{3}{|l|}{ Site of primary disease } \\
\hline Ut & 4 & 22.2 \\
\hline Mt & 9 & 50.0 \\
\hline $\mathrm{Lt}$ & 5 & 27.8 \\
\hline \multicolumn{3}{|l|}{ Differentiation } \\
\hline Well differentiated & 7 & 38.9 \\
\hline Moderately differentiated & 8 & 44.4 \\
\hline Poorly differentiated & 3 & 16.7 \\
\hline
\end{tabular}

Toxicity and dose-finding study

Data on the dose-escalation scheme, DLT, and response are summarized in Table 3 . Only $\geq$ grade 2 toxicity data were collected and reported. Especially for neutropenia and leucopenia, only $\geq$ grade 3 toxicity data were reported. Patient characteristics were well balanced across all dose levels. No treatment-related deaths occurred.
A total of six patients were initially administered the level 1 dose (TXT $30 \mathrm{mg} / \mathrm{m}^{2}$ ). One patient had grade 3 neutropenia lasting 5 days with fever. Three patients were added to the cohort at this dose level. Of the six patients treated at dose level 1, 2 had grade 3 neutropenia with fever lasting 5 days, 1 had grade 2 anemia, and 1 had grade 2 thrombocytopenia. Three patients had grade 2 anorexia, 1 had grade 2 fatigue, 1 had grade 2 nausea, and 2 had grade 2 mucositis. Twenty-four courses of chemotherapy were evaluated. Among the six patients entered at this dose level, five responders were observed.

Three patients were initially enrolled at dose level 2 (TXT $35 \mathrm{mg} / \mathrm{m}^{2}$ ). One patient had grade 3 neutropenia lasting 5 days with fever. Three additional patients were enrolled at this dose level, resulting in six patients being treated at this dose level. One patient had grade 3 neutropenia lasting 5 days with fever, 1 had grade 3 anemia, 1 experienced grade 2 nausea, two experienced grade 2 anorexia, one had grade 1 mucositis, and one patient experienced grade 2 sensory neuropathy. Twenty-seven courses of chemotherapy were administered at dose level 2 . All six of the patients entered at this dose level were responders.

At dose level 3 (TXT $40 \mathrm{mg} / \mathrm{m}^{2}$ ), 1 of 3 patients developed grade 3 toxicity characterized by febrile neutropenia, so three patients were added to the cohort at this dose level. Of the six patients treated at this dose level, one had grade 3 leucopenia, two had grade 3 febrile neutropenia, two experienced grade 2 nausea, two experienced grade 2 anorexia, and one had grade 2 mucositis. This dose (TXT $40 \mathrm{mg} / \mathrm{m}^{2}$ ) was considered the MTD; therefore, the dose of TXT for further phase II studies was determined to be $35 \mathrm{mg} / \mathrm{m}^{2}$ administered biweekly.

Frequency of treatment-related toxicities is summarized in Table 4. Grade 3 leucopenia occurred in one patient (5.6\%). Febrile neutropenia (grade 3) was common and occurred in five patients (27.8\%). Grade 2 nausea occurred in five patients $(27.8 \%)$, and grade $1 / 2$ mucositis occurred in two patients (11.1\%). Alopecia was observed in $100 \%$ of patients. Five courses of chemotherapy $(7 \%)$ were delayed for 1 week due to myelosuppression.

Table 3 Dose-escalation scheme, dose-limiting toxicity, and response

\begin{tabular}{|c|c|c|c|c|c|}
\hline Dose level of docetaxel & Patients & Cycles & DLT & Type & Response \\
\hline $30 \mathrm{mg} / \mathrm{m}^{2}$ & 6 & 24 & 2 & 2 Febrile neutropenia & $2 \mathrm{CR}, 3 \mathrm{PR}, 1 \mathrm{PD}$ \\
\hline $35 \mathrm{mg} / \mathrm{m}^{2}$ & 6 & 27 & 2 & $\begin{array}{l}1 \text { Febrile neutropenia } \\
1 \text { Anemia }\end{array}$ & $3 \mathrm{CR}, 3 \mathrm{PR}$ \\
\hline $40 \mathrm{mg} / \mathrm{m}^{2}$ & 6 & 19 & 3 & $\begin{array}{l}2 \text { Febrile neutropenia } \\
1 \text { Neutropenia }\end{array}$ & $1 \mathrm{CR}, 4 \mathrm{PR}, 1 \mathrm{SD}$ \\
\hline Total & 18 & 70 & 7 & & Response rate: $88.9 \%$ \\
\hline
\end{tabular}

$D L T$ dose-limiting toxicity, $C R$ complete response, $P R$ partial response, $S D$ stable disease, $P D$ progressive disease 
Table 4 Frequency of treatment-related toxicity

\begin{tabular}{|c|c|c|c|}
\hline & \multicolumn{3}{|c|}{ CTCAE ver. 3 common toxicity criteria } \\
\hline & Dose level $1 N=6$ & Dose level $2 N=6$ & Dose level $3 N=6$ \\
\hline \multicolumn{4}{|l|}{ Hematologic } \\
\hline Febrile neutropenia & 2 (grade 3$)$ & 1 (grade 3$)$ & 2 (grade 3$)$ \\
\hline Leucopenia & 0 & 0 & 1 (grade 3$)$ \\
\hline Anemia & 1 (grade 2$)$ & 1 (grade 3$)$ & 0 \\
\hline Thrombocytopenia & 1 (grade 2) & 0 & 0 \\
\hline \multicolumn{4}{|l|}{ Non-hematologic } \\
\hline Anorexia & 3 (grade 2 ) & $2($ grade 2$)$ & 2 (grade 2$)$ \\
\hline Fatigue & 1 (grade 2$)$ & 0 & 0 \\
\hline Mucositis & 0 & $1($ grade 1$)$ & 1 (grade 2$)$ \\
\hline Nausea/vomiting & 1 (grade 2) & 1 (grade 2$)$ & 2 (grade 2$)$ \\
\hline Diarrhea & 0 & 1 (grade 2$)$ & 0 \\
\hline Pericardial effusion & 0 & 0 & 1 (grade 1$)$ \\
\hline \multirow[t]{2}{*}{ Alopecia } & $4($ grade 1$)$ & $3($ grade 1$)$ & $5($ grade 1$)$ \\
\hline & 1 (grade 2$)$ & 3 (grade 2 ) & 1 (grade 2$)$ \\
\hline Edema & 1 (grade 2$)$ & 1 (grade 2) & 1 (grade 2$)$ \\
\hline Sensory neuropathy & 0 & 1 (grade 2$)$ & 0 \\
\hline
\end{tabular}

\section{Tumor response}

Although response to therapy was not the endpoint of this study, patients who had completed at least two cycles of chemotherapy were evaluated for radiographical response. There were six patients with complete response: two patients received two cycles for a locally advanced esophageal cancer and underwent complete resection (histological grades 2 and 3), and four patients received 2-9 cycles for metastatic esophageal cancer (lung, two cases; liver, one case; lung and liver, one case).

Of the 10 patients with partial response, four patients stopped therapy after receiving two or three cycles and underwent surgical curative resection. One patient with stable disease for metastatic esophageal cancer maintained disease stability over five treatment cycles. One patient had documented progressive disease after three cycles with metastatic carcinoma to the liver. The response rate was $88.9 \%$, with six patients $(33.3 \%)$ achieving a complete response and 10 patients $(55.6 \%)$ a partial response. Disease stability and progression were observed in two patients $(11.1 \%)$. No patient discontinued study therapy due to toxicity. Responses were observed at all dose levels, indicating a wide margin of activity for this regimen.

\section{Discussion}

The present study was designed to establish a safe and tolerable dose of TXT (Taxotere; Sanofi-Aventis, Paris, France) when administered in combination with fixed doses of CDDP and 5-FU. TXT is a semi-synthetic taxoid derived from the European yew tree, Taxus baccata. The taxanes enhance polymerization of tubulin into stable microtubule formation and inhibit their tubulin depolymerization by blocking the cell cycle in metaphase, anaphase, and interphase [15]. This inhibition may improve the efficacy of drugs such as CDDP, which are active in all phases of the cell cycle via direct DNA damage. Furthermore, the taxanes increase programmed cell death, and TXT appears to be more potent than paclitaxel in inhibiting angiogenesis [16].

TXT, CDDP, and 5-FU activity occurs by synergistic or non-cross-resistance effects when administered in combination. Previously published studies have shown that the DCF combination demonstrates considerable activity. A $49 \%$ response rate was observed with a DCF combination treating patients with locally advanced esophageal SCC [3].

In the present study, a total of 70 courses of chemotherapy were administered to the 18 patients, and responses were observed at all dose levels. No treatment-related deaths were observed. Toxicity of TXT was encountered at all dose levels, indicating that the pharmacokinetics of this drug may vary in different individuals.

TXT offers favorable outcomes, although it has adverse hematological toxicity. Neutropenia occurs approximately 8-10 days after administration but recovers rapidly. TXT $75-100 \mathrm{mg} / \mathrm{m}^{2}$ every $3-4$ weeks is associated with a quite pronounced neutropenia, with an up to $44 \%$ rate of febrile neutropenia in patients with recurrent ovarian cancer [17]. Takahashi et al. reported that the major toxicity of DCF, repeated every 3 weeks at doses of TXT $50 \mathrm{mg} / \mathrm{m}^{2}$, CDDP $70 \mathrm{mg} / \mathrm{m}^{2}$, and $5-\mathrm{FU} 700 \mathrm{mg} / \mathrm{m}^{2}$, was myelosuppression 
and that the frequencies of grade 3/4 leucopenia and neutropenia in a phase II study were 53.8 and $43.6 \%$, respectively [18].

To minimize toxicity and maximize dose intensity, we elected to investigate a biweekly regimen of TXT with a $40-\mathrm{mg} / \mathrm{m}^{2}$ fixed dose of CDDP and a $400-\mathrm{mg} / \mathrm{m}^{2}$ fixed dose of 5-FU. Compared with the results of a phase I/II study of a TXT-containing regimen for esophageal cancer [18], our regimen included greater TXT dose intensity than the triweekly administration of TXT performed in that study. Divided administration of TXT and CDDP may reduce myelosuppression and neuropathy, while maintaining almost unchanged efficacy.

The median white blood cell and platelet count nadir occurred on day 9 (range 4-18 days), with a median hematological recovery observed by day 12 . Neutropenic fever requiring hospitalization was observed in two patients. Only one patient required a blood transfusion for anemia, and no thrombocytopenia $\geqq$ grade 3 was seen.

The incidence of TXT-specific toxicities, such as neurotoxicity and acute hypersensitivity reactions, was relatively low and did not appear to be a major clinical problem, so a reduction in dose is generally not required. This was probably due to the low cumulative dose (especially with the biweekly dose method) of TXT and CDDP administered.

Fluid retention manifesting as peripheral edema, pleural effusion, or ascites was cumulative in incidence and severity. One patient had grade 4 body-weight gain that required diuretics.

Patients receiving more than $50 \mathrm{mg} / \mathrm{m}^{2}$ of CDDP may suffer nausea and vomiting [19]. Both are frequent side effects caused by administration of CDDP and can be well controlled by administration of granisetron and dexamethasone. Grade 1/2 alopecia was observed in all patients in the present study. Of note, no patient in our study experienced grade 3 or 4 mucositis, likely due to the great care paid to daily supplementation with L-glutamine $8 \mathrm{~g}$, which contributed to the low toxicity profile of this regimen.

Five patients underwent radical surgical resection, with a postoperative mortality of $0 \%$. Pathologically confirmed complete response was documented in one patient. Locoregional disease control was achieved in $88.9 \%$ of the patients in the present study. The results emerging from this phase I study with TXT are particularly encouraging. In the present study, all the 18 patients were diagnosed as squamous cell carcinoma, whereas most esophageal carcinomas in western populations are diagnosed as adenocarcinoma [20]. Given that squamous cell carcinoma and adenocarcinoma are distinct pathologic entity, molecular analyses using cell lines or tissue samples are needed to evaluate the validity that this regimen can be applied to esophageal adenocarcinoma.
In conclusion, the recommended DCF combination dose in the present study was determined to be TXT $35 \mathrm{mg} / \mathrm{m}^{2}$ with CDDP $40 \mathrm{mg} / \mathrm{m}^{2}$ and $5-\mathrm{Fu} 400 \mathrm{mg} / \mathrm{m}^{2}$, administered biweekly. We found that this regimen showed high activation and tolerance such that it could be offered as a candidate component of standard regimens for treating advanced esophageal carcinoma. A phase II study has already been started. Further clinical trials using this combination should be pursued in the treatment of advanced esophageal carcinoma.

Open Access This article is distributed under the terms of the Creative Commons Attribution Noncommercial License which permits any noncommercial use, distribution, and reproduction in any medium, provided the original author(s) and source are credited.

\section{References}

1. Bedenne L, Michel P, Bouché O et al (2007) Chemoradiation followed by surgery compared with chemoradiation alone in squamous cancer of the esophagus: FFCD 9102. J Clin Oncol 25:1160-1168

2. Kranzfelder M, Buchler P, Lange K, Friess H (2010) Treatment options for squamous cell cancer of the esophagus: a systematic review of the literature. J Am Coll Surg 210:351-359

3. Chiarion-Sileni V, Corti L, Ruol A et al (2007) Phase II trial of docetaxel, cisplatin and fluorouracil followed by carboplatin and radiotherapy in locally advanced oesophageal cancer. $\mathrm{Br} \mathrm{J}$ Cancer 96:432-438

4. Cooper JS, Guo MD, Herskovic A et al (1999) Chemoradiotherapy of locally advanced esophageal cancer: long-term followup of a prospective randomized trial (RTOG 85-01) Radiation Therapy Oncology Group. JAMA 281:1623-1627

5. Iizuka T, Kakegawa T, Ide H et al (1992) Phase II evaluation of cisplatin and 5-fluorouracil in advanced squamous cell carcinoma of the esophagus: a Japanese esophageal oncology group trial. Jpn J Clin Oncol 22:172-176

6. De Besi P, Sileni VC, Salvagno L et al (1986) Phase II study of cisplatin, 5-FU, and allopurinol in advanced esophageal cancer. Cancer Treat Rep 70:909-910

7. Bleiberg H, Conloy T, Paillot B et al (1997) Randomised phase II study of cisplatin and 5-fluorouracil (5-FU) versus cisplatin alone in advanced squamous cell oesophageal cancer. Eur J Cancer 33:1216-1220

8. Honda M, Miura A, Izumi Y, Kato T, Ryotokuji T, Monma K, Fujiwara J, Egashira H, Nemoto T 2010 Doxorubicin, cisplatin and fluorouracil combination therapy for metastatic esophageal squamous cell carcinoma. Dis Esophagus 10. Epub ahead of print

9. Yoshida K, Ninomiya M, Hirabayashi N, Takiyama W, Sato Y, Todo S, Terashima M, Gotoh M, Sakamoto J, Nishiyama M (2006) PhaselIstudy of docetaxel and S-1 combination therapy for advanced or recurrent gastric cancer. Clin Cancer Res 12(11):3402-3407

10. Wada Y, Yoshida K, Suzuki T, Mizuiri H, Konishi K, Ukon K, Tanabe K, Sakata Y, Fukushima M (2006) Synergistic effects of docetaxel and S-1 by modulating the expression of metabolic enzymes of 5-fluorouracil in human gastric cancer cell lines. Int J Cancer 119:783-791

11. Hihara J, Yoshida K, Hamai Y, Emi M, Yamaguchi Y, Wadasaki K (2007) PhaseIstudy of docetaxel and 5-fluorouracil with concurrent radiotherapy in patients with advanced esophageal cancer. Anticancer Res 27(4C):597-603 
12. Rigas JR, Dragnev KH, Budis JA (2005) Docetaxel in the treatment of esophageal cancer. Semin Oncol 32:S39-S51

13. Ajani JA, Moiseyenko VM, Tjulandin S et al (2007) Quality of life with docetaxel plus cisplatin and fluorouracil compared with cisplatin and fluorouracil from a phase III trial for advanced gastric or gastroesophageal adenocarcinoma: the V-325 study group. J Clin Oncol 25:3210-3216

14. Therasse P, Arbuck SG, Eisenhauer EA et al (2000) New guidelines to evaluate the response to treatment in solid tumors European Organization for Research and Treatment of Cancer, National Cancer Institute of the United States, National Cancer Institute of Canada. J Natl Cancer Inst 92:205-216

15. Schiff PB, Fant J, Horwitz SB (1979) Promotion of microtubule assembly in vitro by taxol. Nature 277:665-667

16. Grant DS, Williams TL, Zahaczewsky M, Dicker AP (2003) Comparison of antiangiogenic activities using paclitaxel (taxol) and docetaxel (taxotere). Int J Cancer 104:121-129
17. Kaye SB, Piccart M, Aapro M, Francis P, Kavanagh J (1997) Phase II trials of docetaxel (Taxotere) in advanced ovarian cancer-an updated overview. Eur J Cancer 33:2167-2170

18. Takahashi H, Arimura Y, Yamashita K et al (2010) Phase I/II study of docetaxel/cisplatin/fluorouracil combination chemotherapy against metastatic esophageal squamous cell carcinoma. J Thorac Oncol 1:122-128

19. Gralla RJ, Osoba D, Kris MG et al (1999) Recommendations for the use of antiemetics: evidence-based, clinical practice guidelines American Society of Clinical Oncology. J Clin Oncol 17:2971-2994

20. Stelow EB, Mills SE, Jo VY, Carlson DL (2010) Adenocarcinoma of the upper aerodigestive tract. Adv Anat Pathol $17: 262-269$ 